\title{
FORMATO DA INFORMAÇÃO NO CÁLCULO DE PROBABILIDADES POR FUTUROS PROFESSORES DOS PRIMEIROS ANOS
}

\author{
J.A. FERNANDES ${ }^{1 *}$, G. GONÇALVES ${ }^{2}$, P. M. BARROS ${ }^{3}$ \\ ${ }^{1}$ Universidade do Minho, ${ }^{2}$ Instituto Politécnico do Porto, ${ }^{3}$ Instituto Politécnico da Bragança \\ jfernandes@ie.uminho.pt*
}

Artigo submetido em 11/042016/2016 e aceito em 26/06/2019

DOI: $10.15628 /$ holos.2019.8504

\section{RESUMO}

Neste artigo estuda-se o cálculo de probabilidades realizado por futuros professores dos primeiros anos escolares, bem como o formato da informação a que eles recorrem na determinação dessas probabilidades. Participaram no estudo 50 estudantes, futuros professores dos primeiros anos escolares, que na altura se encontravam a frequentar o segundo ano do curso de Licenciatura em Educação Básica numa universidade do norte de Portugal. Aos estudantes foram aplicadas várias tarefas de Probabilidades e Estatística, num contexto de avaliação formal, sendo aqui estudada apenas uma dessas tarefas, a qual envolve vários tipos de probabilidade (probabilidade simples, conjunta e condicionada) e dois formatos de informação (frequências absolutas e frequências relativas). Em termos dos principais resultados obtidos, salienta-se que os futuros professores revelaram um conhecimento limitado de probabilidades e uma preferência clara pelo uso das frequências absolutas no cálculo dessas probabilidades.

PALAVRAS-CHAVE: Probabilidade, formato da informação, futuros professores dos primeiros anos.

\section{INFORMATION FORMAT IN THE CALCULATION OF PROBABILITIES BY PROSPECTIVE PRIMARY SCHOOL TEACHERS}

\begin{abstract}
In this article we study the calculation of probabilities performed by prospective primary school teachers, as well as the format of the information that they use in determining these probabilities. Fifty students, prospective primary school teachers, who were attending the second year of the Basic Education Degree course at a university in the north of Portugal participated in the study. Students were assigned several tasks of Probability and Statistics in a formal
\end{abstract}

evaluation context. Only one of these tasks was studied, which involves several types of probability (simple, joint and conditioned probability) and two information formats (absolute frequencies and relative frequencies). In terms of the main obtained results, it is pointed out that the future teachers revealed a limited knowledge of probabilities and a clear preference for the use of the absolute frequencies in the calculation of these probabilities.

KEYWORDS: Probability, information format, prospective primary school teachers. 


\section{INTRODUÇÃO}

Nas últimas décadas, sobretudo nos países mais desenvolvidos, tem-se assistido a um aprofundamento do ensino da Estatística e das Probabilidades nas escolas, com especial ênfase nos primeiros anos de escolaridade. Em consonância com esses países, as correspondentes alterações curriculares também se fizeram sentir em Portugal, verificando-se, atualmente, que este tema faz parte dos programas de Matemática de todos os anos escolares (Ministério da Educação e Ciência, 2013, 2014).

Ora o facto de os professores terem de ensinar agora estas temáticas implica que eles devem aprofundar o seu conhecimento sobre os respetivos conteúdos já durante a sua formação inicial na universidade. No caso dos futuros professores dos anos escolares iniciais, a questão da sua formação em Estatística e Probabilidades é ainda mais premente pois, no passado recente, porque não tinham de ensinar estes temas, eles também não os estudavam ao longo da sua formação inicial.

A questão do estudo do conhecimento para ensinar surge nos anos oitenta do século passado, com a publicação dos trabalhos de Shulman (1986), assumindo-se como um conhecimento complexo e multifacetado. Na sequência destes estudos, outros autores (e.g., Hill, Ball \& Schilling, 2008; Godino, Batanero \& Font, 2007) estabeleceram diferentes categorias desse conhecimento. Porém, para além de outras, identificam-se duas principais categorias do conhecimento para ensinar comuns a todos esses autores: o conhecimento do conteúdo e o conhecimento pedagógico do conteúdo.

No presente estudo indagamos futuros professores dos primeiros anos sobre o conhecimento do conteúdo, neste caso de probabilidades, e o formato da informação por eles usada na determinação de probabilidades. No caso do conhecimento do conteúdo, Hill et al. (2008) distinguem várias categorias desse conhecimento, de entre as quais se salienta, neste estudo, o conhecimento avançado do conteúdo, que é um conhecimento que vai além daquele que o professor terá de ministrar aos seus alunos. Por outro lado, no enunciado da tarefa são fornecidos dados que permitem aos futuros professores optarem por recorrer a dois formatos de informação na determinação das probabilidades requeridas, isto é, recorrer às frequências relativas em percentagem (dadas no enunciado) ou às correspondentes frequências absolutas, que deverão ser previamente determinadas.

Nas próximas secções do artigo trataremos do enquadramento teórico do estudo, da metodologia seguida no estudo, da determinação das probabilidades pelos futuros professores e das principais conclusões e implicações do estudo.

\section{ENQUADRAMENTO TEÓRICO}

Tal como antes referimos, o conhecimento para ensinar é um conhecimento complexo que se desdobra em múltiplas categorias. No caso de Shulman (1986), que foi pioneiro no estudo desse conhecimento, ele identificou três grandes categorias: o conhecimento do conteúdo, que se refere ao conhecimento do que se vai ensinar; o conhecimento pedagógico do conteúdo, relativo às formas como o professor representa e formula o seu conhecimento do conteúdo quando ensina; e o 
conhecimento do currículo, que se refere ao conhecimento dos programas escolares, dos materiais de ensino e das indicações neles existentes.

Na sequência de Shulman, também Hill et al. (2008) distinguiram duas principais categorias de conhecimento relevantes para o professor de Matemática: o conhecimento matemático e o conhecimento pedagógico do conteúdo. A categoria do conhecimento matemático desdobra-se ainda em três componentes: o conhecimento comum do conteúdo, que se refere ao conhecimento que alguém tem de mobilizar para resolver problemas matemáticos; o conhecimento especializado do conteúdo, que diz respeito ao conhecimento especial do professor para planificar e desenvolver sequências de ensino; e o conhecimento matemático avançado, que se refere aos aspetos mais avançados do conteúdo matemático, incluindo também aspetos históricos e filosóficos.

A categoria do conhecimento pedagógico do conteúdo também se desdobra em três componentes: o conhecimento do conteúdo e os estudantes, que é relativo ao conhecimento sobre como os estudantes pensam, concetualizam ou aprendem um conteúdo; o conhecimento do conteúdo e o ensino, que resulta da integração do conhecimento do conteúdo matemático com o conhecimento sobre o ensino desse conteúdo; e o conhecimento do currículo, que se refere ao conhecimento das diretrizes curriculares, orientações, fins e motivações das mesmas, materiais curriculares e sequencialização do tema nos diferentes níveis escolares.

Integrando, ampliando e detalhando os modelos anteriores, Godino, Batanero e Font (2007) e Godino (2009) propõem várias facetas para analisar os processos de estudo e instrução da Matemática: a faceta epistémica, que se refere aos conhecimentos matemáticos relativos ao contexto institucional em que se realiza o processo de aprendizagem, e envolvendo os vários objetos matemáticos primários: problemas, linguagens, procedimentos, definições e conceitos, propriedades e argumentos; a faceta cognitiva, relativa aos conhecimentos pessoais dos estudantes e à progressão das aprendizagens; a faceta afetiva, que diz respeito aos estados afetivos (atitudes, emoções, crenças, valores) de cada aluno em relação aos objetos matemáticos e ao processo de estudo seguido; a faceta mediacional, relativa aos recursos de ensino usados, particularmente os tecnológicos, e à atribuição do tempo às distintas ações e processos; a faceta interacional, referente aos padrões de interação entre o professor e os alunos e sua sequencialização orientada para a fixação e negociação de significados; e a faceta ecológica, em que se enfatiza o sistema de relações com o contexto social, político e económico.

Considerando as diferentes categorias do conhecimento para ensinar, discutidas anteriormente, no presente estudo focamo-nos no conhecimento do conteúdo dos futuros professores (Shulman (1986), no conhecimento matemático avançado (Hill et al., 2008), e nas facetas epistémica e cognitiva (Godino 2009; Godino et al., 2007). Seguidamente iremos rever alguns estudos envolvendo o conhecimento de probabilidades de futuros professores dos primeiros anos.

Para além de probabilidades simples, no presente estudo é requerido aos futuros professores que determinem probabilidades conjuntas e probabilidades condicionadas, conceitos estes que, segundo vários autores, se encontram entre aqueles que mais ideias erradas despoletam nos estudantes (e.g., Polaki, 2005; Tarr \& Lannin, 2005; Watson \& Moritz, 2002).

Num estudo envolvendo futuros professores do ensino primário, Contreras, Estrada, Díaz e Batanero (2010) concluíram que eles tiveram muitas dificuldades na determinação da 
probabilidade condicionada e conjunta, tendo alguns aderido a raciocínios falaciosos. Também num estudo com futuros professores dos primeiros anos de escolaridade, envolvendo conteúdos mais diversificados, Fernandes, Viseu e Gea (2016) constataram que os futuros professores, excetuando o caso da probabilidade simples, em que foram mais sucedidos, demonstraram um desempenho limitado nos itens sobre a definição de acontecimentos certos e sobre a probabilidade condicionada, e um desempenho ainda mais limitado na probabilidade conjunta.

Pesquisando a origem das dificuldades em probabilidade conjunta e condicionada de futuros professores dos primeiros anos, Fernandes, Batanero, Correia e Gea (2014) constataram que eles combinaram erradamente os valores da probabilidade conjunta, destacando-se a aplicação da operação de adição em vez da de multiplicação, consideraram apenas a probabilidade de uma das duas ordens possíveis (não atendo à ordem), determinaram o valor de probabilidade de apenas um dos acontecimentos implicados, ignoraram o acontecimento condicionante, confundiram probabilidade condicionada com probabilidade conjunta, consideraram indevidamente a reposição ou não reposição e determinaram casos favoráveis e casos possíveis sem os combinarem.

Também no contexto de professores chilenos do ensino primário em exercício, sobre o conhecimento didático-matemático em Probabilidades, Alsina e Vásquez (2016) verificaram que esses professores revelaram vários dificuldades e erros e aderiram a heurísticas e enviesamentos probabilísticos. Face a estas dificuldades, os autores advogam a necessidade de aprofundar o conhecimento didático-matemático destes professores, designadamente nas três componentes do conhecimento matemático de Hill et al. (2008), que foram antes referidas.

Num estudo mais recente, com futuros professores semelhantes àqueles que participam no presente estudo, Fernandes e Gea (2018) constataram que eles revelaram muitas dificuldades em dois itens de probabilidade conjunta, com menos de metade dos estudantes a responderem corretamente e observando-se conflitos semióticos de reposição, de não ordem, de determinação de probabilidades simples, de adicionar probabilidades em vez de multiplicar e de afirmar a maior probabilidade de extrair duas bolas brancas pelo facto de existirem mais bolas brancas no saco. Para além destes dois itens, focados no conhecimento do conteúdo (faceta epistémica), os mesmos futuros professores também foram inquiridos sobre a avaliação de várias respostas de hipotéticos alunos dos primeiros anos às mesmas questões, portanto itens centrados no conhecimento do conteúdo e os estudantes (faceta cognitiva). Apesar de se ter verificado uma melhoria na classificação das respostas dos alunos em corretas e incorretas, novamente foram poucos os futuros professores que identificaram os conflitos semióticos inerentes às respostas incorretas dos hipotéticos alunos.

Num estudo ainda mais recente, Vásquez e Alsina (2019) concluíram que o conhecimento especializado de professores primários chilenos em exercício era claramente insuficiente para ensinar Probabilidades aos seus alunos. Ora, estas limitações do conhecimento especializado refletiram-se em todas as facetas do conhecimento estabelecidas por Godino (2009), designadamente na faceta epistémica, na faceta cognitiva, na faceta afetiva, na faceta mediacional, na faceta instrucional e na faceta ecológica.

Dos estudos antes referidos, e mais se poderiam apresentar, conclui-se que o conhecimento dos futuros professores dos primeiros anos para ensinar probabilidades é limitado, mais ainda se nos referirmos ao conhecimento matemático avançado, que estudamos neste artigo. 
Para além dos aspetos concetuais e procedimentais, também os aspetos representacionais influenciam o desempenho e os processos de resolução adotados pelos estudantes. Neste caso, Gigerenzer e Hoffrage (1995) e Hoffrage, Gigerenzer, Krauss e Martignon (2002) concluíram que o formato de representação da informação condiciona o sucesso dos sujeitos na resolução de problemas envolvendo a probabilidade condicionada e a inferência bayesiana. Gigerenzer e Hoffrage (1995) mostraram, com vários estudos, que se facilita o raciocínio dos sujeitos alterando a representação na forma de probabilidades e frequências relativas (eventualmente em percentagem) para frequências absolutas, a que estes autores chamam frequências naturais. Para Hoffrage et al. (20002) as probabilidades e as frequências relativas tornam a inferência bayesiana computacionalmente mais complexa do que as frequências naturais, até porque o cálculo da probabilidade condicionada $P(H \mid D)$ é mais simples quando a informação é dada em frequências naturais do que quando é dada em probabilidades ou frequências relativas.

No presente estudo é fornecida informação em diferentes formatos, concretamente sob a forma de frequências absolutas e frequências relativas (em percentagem), podendo os estudantes optar por usarem as frequências relativas dadas para determinar as várias probabilidades pedidas ou começar por determinarem as correspondentes frequências absolutas para depois calcularem as respetivas probabilidades solicitadas.

\section{METODOLOGIA}

Nesta investigação estuda-se a realização de futuros professores dos primeiros anos em diferentes tipos de probabilidade, em termos de respostas e de processos de resolução, analisando-se nos processos de resolução dos estudantes também o formato da informação por que eles optaram na determinação das respetivas probabilidades.

Participaram no estudo 50 estudantes (designados por $E i$, com $i=1,2, \ldots, 50$ ) de uma universidade do norte de Portugal, que se encontravam a frequentar a unidade curricular de Probabilidades e Estatística, que faz parte do plano de estudos do 2. ano do curso de Licenciatura em Educação Básica. À entrada na universidade, estes estudantes tinham uma formação matemática muito variada, o que explica as diferentes perceções sobre as suas dificuldades nas disciplinas de matemática que tinham frequentado, até à altura, na universidade. Precisamente, $72 \%$ dos estudantes afirmou ter muita dificuldade ou dificuldade, enquanto $28 \%$ afirmou ter pouca dificuldade ou não ter dificuldade, o que significa que quase três em cada quatro estudantes afirmaram ter dificuldade ou muita dificuldade nas disciplinas de matemática.

A recolha de dados aconteceu após a lecionação da disciplina de Probabilidades e Estatística e consistiu na aplicação de várias tarefas de Probabilidades e Estatística, em contexto de avaliação formal. Dessas tarefas, estudamos aqui apenas uma, relativa ao tema Probabilidades, que se apresenta na Figura 1.

Numa escola, com 300 alunos, sabe-se que $40 \%$ são raparigas. Além disso, sabe-se ainda que $70 \%$ das raparigas não pratica esgrima e $80 \%$ dos rapazes pratica esgrima.

Escolhendo um aluno ao acaso,

a) Qual a probabilidade de praticar esgrima?

b) Qual a probabilidade de ser rapariga e não praticar esgrima? 
c) Qual a probabilidade de ser rapariga, sabendo que não pratica esgrima?

d) Qual a probabilidade de praticar esgrima, sabendo que é rapaz?

Figura 1: Tarefa proposta aos estudantes.

Observando a tarefa, constata-se que ela é constituída por quatro itens, dos quais em a) é pedido ao estudante que determine uma probabilidade simples, em b) uma probabilidade conjunta e em c) e d) uma probabilidade condicionada.

Deve notar-se que o dado "300 alunos", que aparece no enunciado, é redundante pois a tarefa pode ser resolvida apenas com as percentagens dadas. Contudo, essa informação permite aos estudantes recorrer a outros métodos para resolverem a tarefa, o que constitui também, para além do cálculo das probabilidades, um objetivo deste estudo. Mais especificamente, com esse dado, correspondente ao número total de alunos da escola, os estudantes poderão determinar as frequências absolutas e usá-las na determinação das respetivas probabilidades, resultando daí que eles poderão determinar as frequências absolutas ou recorrer às frequências relativas em percentagem dadas, ou seja, poderão recorrer a informação em diferentes formatos para determinar as probabilidades pedidas.

Por último, em termos de análise de dados, estudamos as respostas dados pelos estudantes quer em termos do tipo de resposta (correta e incorreta) quer em termos dos processos de resolução. No caso dos processos de resolução, recorremos à análise de conteúdo para categorizar os raciocínios usados pelos estudantes nas suas resoluções, o que foi efetuado aquando da análise das suas respostas, na secção seguinte. Adicionalmente, na análise dos processos de resolução discriminámos entre o uso de frequências relativas e a determinação e uso de frequências absolutas na determinação das respetivas probabilidades, tendo em visto distinguir o formato da informação a que os estudantes recorreram.

\section{DETERMINAÇÃO DAS PROBABILIDADES PELOS FUTUROS PROFESSORES}

Nesta secção analisam-se as resoluções apresentadas pelos estudantes à tarefa proposta, considerando o tipo de resposta (correta e incorreta) e as estratégias adotadas para obter essas respostas, incluindo a identificação do formato da informação usada, isto é, frequências relativas (em percentagem) versus frequências absolutas.

\subsection{Respostas}

$\mathrm{Na}$ Tabela 1 apresentam-se as frequências absolutas (em \%) dos tipos de respostas (corretas e incorretas) apresentadas pelos estudantes em cada um dos quatro itens da tarefa, bem como de não respostas. Recorde-se que os itens envolvem vários tipos de probabilidade, avaliando-se no item a) uma probabilidade simples, no item b) uma probabilidade conjunta e nos itens c) e d) probabilidades condicionadas.

Tabela 1: Frequências absolutas (em \%) dos diferentes tipos de resposta em cada um dos itens da tarefa.

\begin{tabular}{l|c|c|c|c}
\hline \multirow{2}{*}{ Tipo de resposta } & \multicolumn{4}{|c}{ Item } \\
\cline { 2 - 5 } & a) & b) & c) & d) \\
\hline Correta & $33(66)$ & $30(60)$ & $24(48)$ & $23(46)$ \\
\hline
\end{tabular}




\begin{tabular}{l|l|l|l|l}
\hline Incorreta & $17(34)$ & $19(38)$ & $19(38)$ & $21(42)$ \\
\hline Não resposta & - & $1(2)$ & $7(14)$ & $6(12)$ \\
\hline
\end{tabular}

Por observação da Tabela 1 verifica-se que mais de metade dos estudantes responderam corretamente aos itens $a$ ) e b), enquanto menos de metade responderam corretamente aos itens c) e d), o que se deve, fundamentalmente, ao aumento das não respostas nestes dois itens. Já em termos de variação, verifica-se que a frequência de respostas corretas foi maior no item de probabilidade simples (item a), diminui no item de probabilidade conjunta (item b) e diminuiu mais ainda nos itens de probabilidade condicionada (itens $\mathrm{c}$ e d).

Tal como aconteceu neste estudo, também em outros estudos anteriores (e.g., Fernandes, 2018; Fernandes et al., 2014; Fernandes et al., 2016) se verificou que os estudantes, também futuros professores dos primeiros anos, tiveram mais dificuldades na probabilidade conjunta e na probabilidade condicionada do que na probabilidade simples. Todavia, contrariamente ao que se verificou no estudo de Fernandes et al. (2014), neste estudo os estudantes tiveram mais dificuldades na probabilidade condicionada do que na probabilidade conjunta, o que pode deverse ao facto de no presente estudo a probabilidade conjunta poder ser transformada numa probabilidade simples, tal como veremos adiante.

Seguidamente, iremos caracterizar as estratégias adotadas pelos estudantes na resolução de cada um dos quatro itens da tarefa, referindo também o formato da informação usada.

\subsection{Estratégias de resolução}

\subsubsection{Item a): probabilidade simples}

Embora não fosse necessário determinar as frequências absolutas, pois podia-se calcular a probabilidade a partir das frequências em percentagem dadas, a maioria estudantes dos estudantes (84\%) começou por determinar as frequências absolutas, para depois determinar a respetiva probabilidade. Esta abordagem conduziu à resposta correta para a maioria destes estudantes (62\%), enquanto as respostas erradas (22\%) resultaram de erros de cálculo na determinação das frequências. Na Figura 2 apresenta-se uma abordagem recorrendo às frequências absolutas.

Total: 300 alunos

$40 \%$ raparigas $\Rightarrow 120$ raparigas

Das 120 raparigas, $70 \%$ não preatica esgrima $\Rightarrow 36$ raparigas praticam esgreima

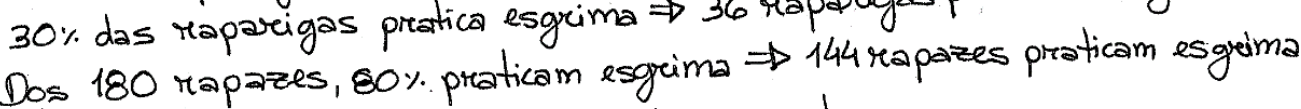

Saberros que no total $36+144=180$ alunos preaticam esgrima

$P($ praticar esgrima $)=\frac{180}{300}=0,6$

Figura 2: Resposta dada pelo estudante E1 ao item a).

Na resolução do estudante E1 constatamos que ele começou por determinar o número de raparigas e de rapazes que praticam esgrima, de seguida adicionou os valores obtidos e, 
finalmente, definiu a razão entre a soma obtida e o número de alunos da escola. Dos estudantes que adotaram esta estratégia, verificou-se, ainda, que quatro organizaram as frequências absolutas numa tabela de dupla entrada.

Diferentemente, foram cinco os estudantes (10\%) que recorreram às frequências relativas para determinarem a probabilidade pedida, dos quais dois responderam erradamente porque cometeram erros ao operarem com essas frequências. Destes estudantes, dois organizaram as frequências relativas numa tabela de dupla entrada e um num diagrama de árvore. Na Figura 3 apresenta-se o diagrama de árvore apresentado pelo respetivo estudante.

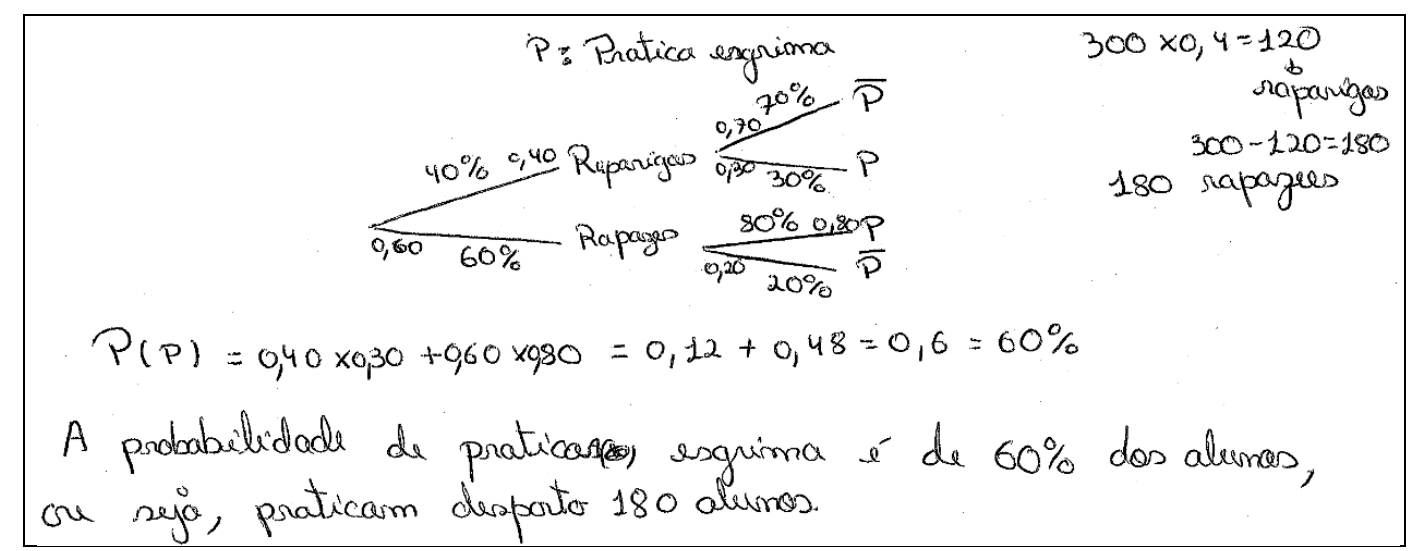

Figura 3: Resposta dada pelo estudante E40 ao item a).

Nesta resolução, o estudante E40 ainda determinou o número de raparigas e de rapazes, mas essa informação não foi usada para calcular a probabilidade pedida. Em vez das frequências absolutas, este estudante usou e operou com as frequências relativas obtidas diretamente do enunciado, tendo adicionado duas probabilidades conjuntas para obter a probabilidade pretendida. Consequentemente, comparativamente com o uso das frequências absolutas, verificase que o recurso às frequências relativas (em percentagem) implicou o recurso a outros tipos de probabilidade.

Observaram-se ainda mais três respostas erradas, das quais duas apresentam um valor da probabilidade superior a um e outra apresenta apenas a representação simbólica dos acontecimentos numa tabela de dupla entrada.

\subsubsection{Item b): probabilidade conjunta}

Tal como no item a), também neste item a maioria dos estudantes (68\%) determinou e/ou operou com as frequências absolutas. Destes estudantes, a maior parte (56\%) respondeu corretamente e apenas poucos estudantes (12\%) responderam incorretamente ao cometer erros de cálculo. Neste item, apenas um estudante organizou as frequências absolutas numa tabela de dupla entrada, embora o tenha feito de forma errada.

Analogamente, também, neste item, foram poucos os estudantes (6\%) que recorreram às frequências relativas para determinar a probabilidade conjunta. Destes estudantes, um organizou as frequências relativas corretamente numa tabela de dupla entrada, tal como se mostra na Figura 4. 


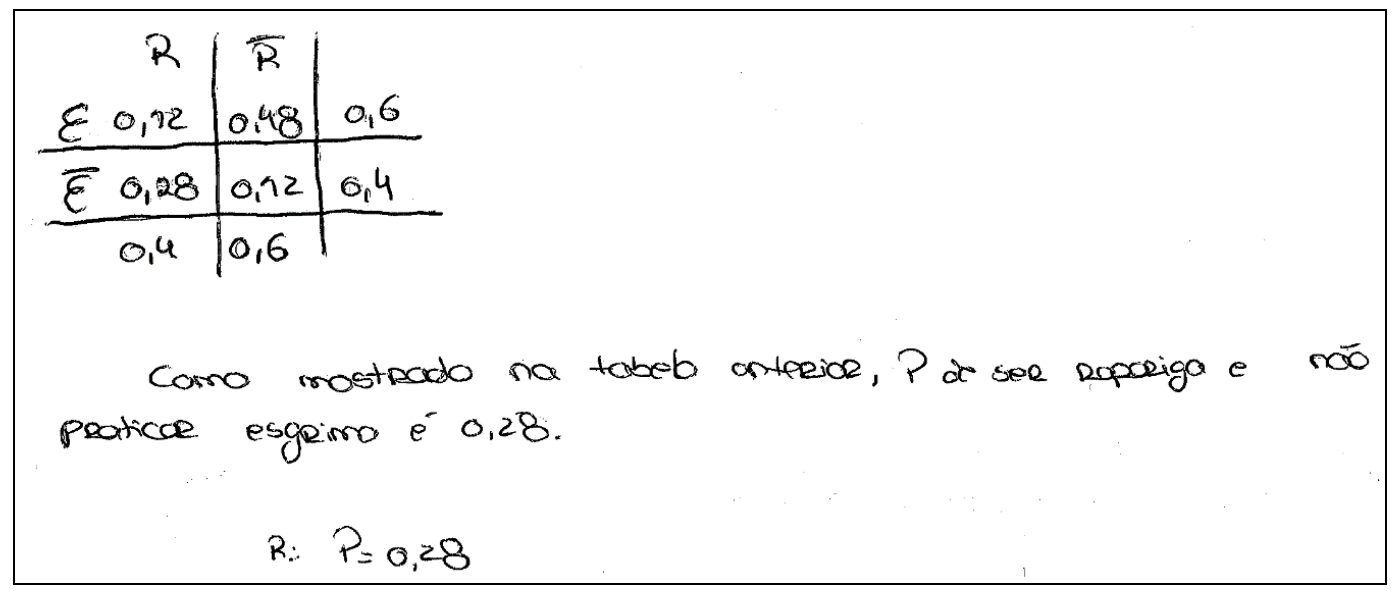

Figura 4: Resposta dada pelo estudante E32 ao item b).

Representando por $E$ o acontecimento "praticar esgrima" e por $R$ o acontecimento "ser rapariga", verifica-se que o estudante E32 transformou em frequências relativas as frequências em percentagem dadas no enunciado e, a partir delas, determinou as outras frequências relativas de modo a completar a tabela de dupla entrada. Seguidamente, leu diretamente da tabela o valor da probabilidade pedida.

Para além dos erros de cálculo, outras respostas incorretas deveram-se a que quatro estudantes apenas traduziram simbolicamente a probabilidade pedida, seis confundiram a probabilidade conjunta com a probabilidade condicionada, cinco apresentaram um dado do enunciado, dois consideraram independentes os acontecimentos da probabilidade conjunta e quatro interpretaram erradamente a probabilidade pedida, como seja, considerar o número de raparigas que praticam esgrima (dois), o número de raparigas que não praticam esgrima (um) e determinar a probabilidade das raparigas que praticam esgrima (um). Conclui-se, assim, que três estudantes apresentaram para a probabilidade pedida valores superiores a um. Na Figura 5 apresenta-se um exemplo em que o estudante confundiu a probabilidade conjunta com a probabilidade condicionada.

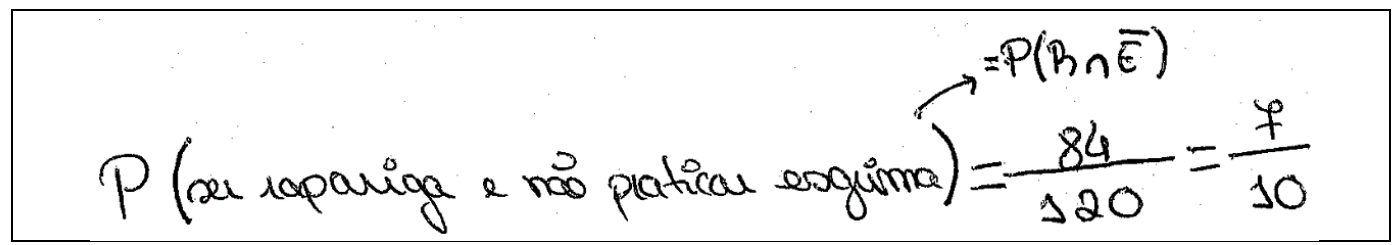

Figura 5: Resposta dada pelo estudante E17 ao item b).

O estudante E17 começou por determinar o número de raparigas que não praticam esgrima, para depois determinar a razão entre esse número e o número total de raparigas. Deste modo, diferentemente do que refere, o estudante calculou a probabilidade de não praticar esgrima sabendo que é rapariga, recorrendo, para tal, à restrição do espaço amostral.

\subsubsection{Item c): probabilidade condicionada}

Novamente, a abordagem mais frequente consistiu em determinar e/ou operar com as frequências absolutas para determinar a probabilidade condicionada (66\%), tal como se verificou com as probabilidades anteriores. Nesta abordagem, quase tantos estudantes responderam corretamente (34\%) como incorretamente (32\%), neste último caso devido a erros de cálculo. 
Tal como nos itens anteriores, poucos estudantes (12\%) determinaram e/ou operaram com as frequências relativas para determinarem a respetiva probabilidade condicionada. Destes estudantes, dois erraram ao determinar as probabilidades que intervêm na fórmula da probabilidade condicionada, um construiu um diagrama de árvore e outro uma tabela de dupla entrada.

Na determinação da probabilidade condicionada, os estudantes adotaram duas estratégias: aplicaram a fórmula da probabilidade condicionada e restringiram o espaço amostral. A aplicação da fórmula da probabilidade condicionada foi a abordagem mais usada pelos estudantes (50\%), dos quais mais responderam corretamente (34\%), tendo as respostas incorretas (16\%) sido devidas a erros no cálculo das probabilidades intervenientes na fórmula. Na Figura 6 pode ver-se uma resolução em que se recorre à formula da probabilidade condicionada.

$\begin{array}{ll}P(M \mid \bar{P})=\frac{P(M \cap \bar{P})}{P(\bar{P})} & P(M \cap \bar{P})=\frac{84}{300}=0,28 \\ P(M \mid \bar{P})=\frac{0,28}{0,4}=0,7 & P(\bar{P})=\frac{120}{300}=0,4\end{array}$

Figura 6: Resposta dada pelo estudante E44 ao item c).

Considerando os acontecimentos $\mathrm{M}$ : ser rapariga e P: praticar esgrima, o estudante E44 recorreu às probabilidades, que calculou a partir das frequências absolutas antes determinadas, substituiu-as na fórmula da probabilidade condicionada, obtendo assim a probabilidade pretendida.

A abordagem da restrição do espaço amostral foi adotada por menos estudantes (22\%), dos quais alguns (8\%) obtiveram uma probabilidade incorreta ao não restringirem adequadamente o espeço amostral. Na Figura 7 apresenta-se um exemplo de resposta obtida através da restrição do espaço amostral.

$$
P(\text { sen napariga } / \text { nod partica esgnima })=\frac{84}{120}=\frac{7}{10} \text {. }
$$

Figura 7: Resposta dada pelo estudante E6 ao item c).

$\mathrm{O}$ estudante $\mathrm{E} 6$, restringindo o universo amostral ao número de alunos que não praticam esgrima, determinou a fração do número de raparigas que não praticam esgrima no total de alunos que não praticam esgrima.

Além dos erros já referidos, oito estudantes confundiram a probabilidade condicionada com a probabilidade conjunta, tendo um desses estudantes apresentado o número de raparigas que praticam esgrima como sendo a probabilidade pedida, tratando-se, obviamente, de um valor superior a um. Na Figura 8 apresenta-se uma resolução em que o estudante confunde a probabilidade condicionada com a probabilidade conjunta.

$$
P\left(\text { Rapariga/náo pratica esgrima) }=\frac{84}{300}=\frac{42}{150}=\frac{21}{75}=\frac{7}{25}\right.
$$

Figura 8: Resposta dada pelo estudante E29 ao item c). 
O estudante E29 recorreu às frequências absolutas, determinadas em itens anteriores, para determinar a probabilidade conjunta, errando ao considerar todo o espaço amostral e não a restrição aos alunos que não praticam esgrima.

\subsubsection{Item d): probabilidade condicionada}

Tal como no item anterior, em que também se pedia uma probabilidade condicionada, verificou-se que a maioria dos estudantes (58\%) determinou e/ou operou com frequências absolutas para determinar a probabilidade condicionada pretendida. Destes estudantes, mais (34\%) responderam corretamente do que erradamente (24\%), devendo-se as dificuldades dos estudantes, fundamentalmente, a erros de cálculo na determinação das respetivas frequências ou probabilidades. Na Figura 9 mostra-se uma resolução envolvendo frequências absolutas.

\begin{tabular}{|c|c|}
\hline$P\left(E \mid \theta^{\prime}\right)=\frac{P\left(E \cap 0^{3}\right)}{P\left(\theta^{3}\right)}=$ & $\begin{array}{l}\sigma^{5}: 17 a p a z \\
100 \%-300 \text { alunos } \\
60 \%-x\end{array}$ \\
\hline$=\frac{\frac{24}{300}}{\frac{180}{300}}=\frac{7200}{5400}=\frac{2}{15}$ & rapazes $n=\frac{60 \times 300}{100}=180$ \\
\hline
\end{tabular}

Figura 9: Resposta dada pelo estudante E9 ao item d).

Na sua resolução, o estudante E9 determina incorretamente a probabilidade de ser rapaz e praticar esgrima (numerador da fração) e comete um erro de cálculo ao considerar o valor 5400 em vez do valor 54000 na divisão das frações.

Já no caso das frequências relativas foram poucos os estudantes (8\%) que as usaram para determinar a probabilidade condicionada, tendo um deles respondido incorretamente porque não determinou ou identificou corretamente as frequências relativas intervenientes no cálculo da probabilidade condicionada.

Considerando agora a determinação da probabilidade condicionada, verificou-se que a maior parte dos estudantes recorreu à fórmula da probabilidade condicionada (42\%) e outros recorreram à restrição do espaço amostral (28\%). No caso da aplicação da fórmula da probabilidade condicionada, verificou-se que mais estudantes $(28 \%)$ responderam corretamente do que incorretamente (14\%), devendo-se as respostas incorretas, fundamentalmente, a erros de cálculo das frequências. Também na determinação da probabilidade condicionada através da restrição do espaço amostral se obtiveram mais respostas corretas (18\%) do que incorretas (10\%), estas últimas devido a erros no cálculo ou na identificação das respetivas frequências. Na Figura 10 apresenta-se uma resolução através da restrição do espaço amostral.

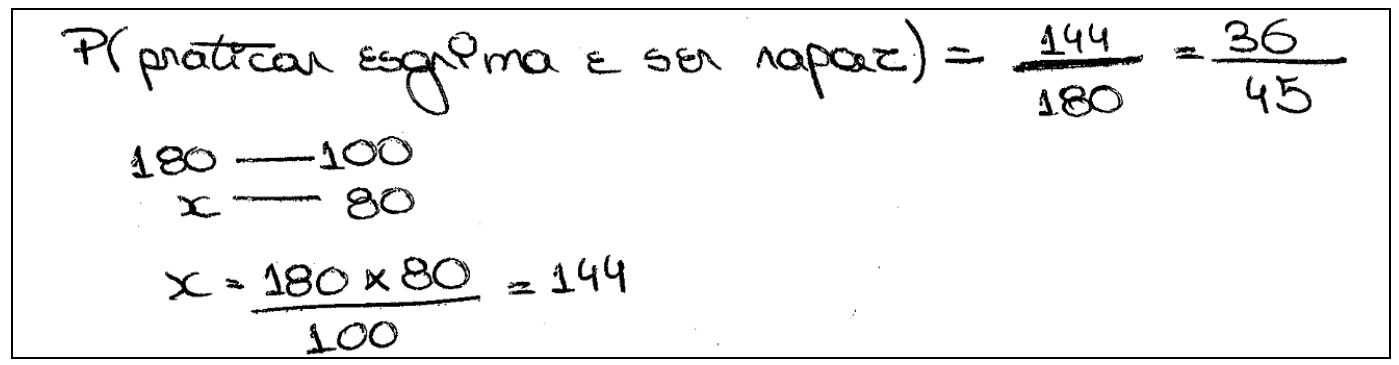


Figura 10: Resposta dada pelo estudante E47 ao item d).

O estudante E47 considerou a restrição do espaço amostral ao conjunto dos rapazes, seguidamente determinou o número de rapazes que praticam esgrima e, finalmente, estabeleceu a razão que define corretamente a probabilidade condicionada pretendida.

Para além das respostas incorretas antes referidas, cinco estudantes confundiram a probabilidade condicionada com a probabilidade conjunta, um estudante indicou um dado do enunciado, outro indicou o número de rapazes que praticam esgrima e outro considerou na fórmula o número de rapazes que não praticam esgrima. Salienta-se ainda que três estudantes apresentaram um valor superior a um para a probabilidade pedida. Na Figura 11 apresenta-se uma resolução em que o valor da probabilidade é superior a um.

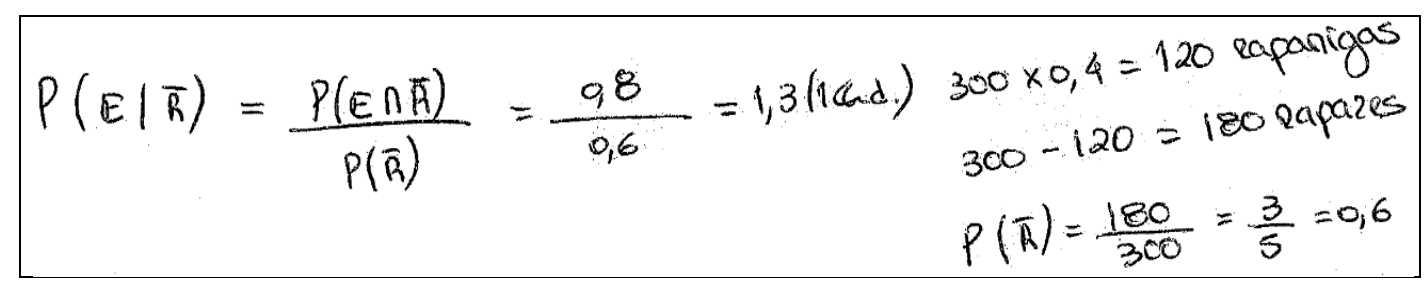

Figura 11: Resposta dada pelo estudante E25 ao item d).

Ao aplicar a fórmula da probabilidade condicionada, o estudante E25 considera erradamente a $P(E \cap \bar{R})$ como sendo a percentagem de rapazes que praticam esgrima, obtendo assim um valor superior a um para a probabilidade condicionada. Face a esse valor, o estudante aceita-o sem qualquer questionamento. Observa-se ainda que o estudante determina corretamente a $P(\bar{R})$, recorrendo agora às frequências relativas que calculou antes.

\section{CONCLUSÃO E IMPLICAÇÕES}

O conhecimento de probabilidades dos estudantes, futuros professores dos primeiros anos, especialmente numa vertente de conhecimento matemático avançado (Hill et al., 2008), ou seja, na faceta epistémica (Godino, 2009), revelou-se um tanto limitado. Em termos da percentagem de respostas corretas, observou-se uma variação entre 66\% (máximo) e 46\% (mínimo), correspondendo a uma média de $55 \%$ na globalidade dos quatro itens da tarefa. Adicionalmente, verifica-se que a percentagem de respostas corretas varia também com o tipo de probabilidade, sendo máxima para a probabilidade simples e diminuindo na probabilidade conjunta e, mais ainda, na probabilidade condicionada. Este conhecimento limitado de Probabilidades tem sido verificado em outros estudos antes realizados (e.g., Contreras et al., 2010; Fernandes \& Gea, 2018; Fernandes et al. 2014; Fernandes et al., 2016; Fernandes et al., 2018).

Em termos das respostas incorretas, para além dos erros de cálculo cometidos pelos estudantes na determinação das frequências e/ou na determinação das probabilidades, com uma maior incidência, destacam-se a confusão entre probabilidade conjunta e probabilidade condicionada e a atribuição de um valor superior a um para valor de probabilidade. No caso da confusão entre probabilidade conjunta e condicionada, ela aparece documentada em muitos estudos (e.g., Estrada \& Díaz, 2006; Fernandes, 2013; Pollatsek, Well, Konold \& Hardiman, 1987) e a 
atribuição à probabilidade de um valor superior a um significa a violação de um dos princípios básicos da noção de probabilidade.

Um outro resultando relevante do presente estudo consistiu no uso preferencial, pelos estudantes, das frequências absolutas na determinação das várias probabilidades, tendo tal uso variado entre $84 \%$ (máximo) e 58\% (mínimo), correspondendo a uma média de 69\% na globalidade dos quatro itens da tarefa. Conclui-se, assim, que cerca de dois em cada três alunos determinaram previamente as frequências absolutas para, depois, as usarem na determinação das respetivas probabilidades. Este resultado confirma, ainda que implicitamente, as conclusões de Gigerenzer e Hoffrage (1995) e de Hoffrage et al. (2002) quando afirmam que os sujeitos têm mais facilidade em efetuar inferências bayesianas e determinar probabilidades condicionadas ao usar as frequências absolutas (a que chamam frequências naturais) do que quando usam probabilidades ou frequências relativas. Diferentemente dos estudos antes referidos, em que a informação das tarefas era dada num dos formatos, frequências absolutas ou frequências relativas, no presente estudo a tarefa envolvia os dois formatos, podendo os estudantes optar por usar as frequências relativas dadas no enunciado ou determinar as frequências absolutas.

Ainda em termos de estratégias de resolução assinala-se neste estudo a determinação das probabilidades condicionadas por dois processos: usando a fórmula da probabilidade condicionada e recorrendo à restrição do espaço amostral. Particularmente no caso da estratégia de restrição do espaço amostral salienta-se que a determinação da probabilidade condicionada é muito semelhante à determinação da probabilidade simples, considerando agora, não o espaço amostral, mas a adequada restrição desse espaço. Ora, o facto de tratar-se de uma abordagem mais simples ao conceito de probabilidade condicionada, torna-a especialmente adequada para explorar o conceito com os alunos dos primeiros anos escolares. Nesta abordagem à probabilidade condicionada, Correia e Fernandes (2013) verificaram que mais de metade dos alunos do 9. ano, que participaram num estudo, apresentaram respostas corretas em mais de dois terços dos itens. Já Fernandes, Correia e Contreras (2013) relatam resultados ainda mais favoráveis, com mais de dois terços dos alunos do 9. . ano a apresentarem respostas corretas.

Do presente estudo podem extrair-se três principais implicações em termos do ensino e da aprendizagem de Probabilidades nos primeiros anos escolares. A primeira refere-se à necessidade de aprofundar o conhecimento para ensinar Probabilidades dos futuros professores dos primeiros anos, pois só assim terão as condições necessárias para implementar um ensino adequado. A segunda consiste em começar a abordagem das probabilidades pelas frequências absolutas e só depois recorrer a outras formas de representação da informação. Por último, abordar com os futuros professores na sua formação inicial a probabilidade condicionada a partir da restrição do espaço amostral, de modo a poderem fazê-lo também com os seus futuros alunos dos primeiros anos.

\section{REFERÊNCIAS}

Alsina, A., \& Vásquez, C. (2016). Análisis de los conocimientos probabilísticos del profesorado de Educación Primaria. Revista digital Matemática, Educación e Internet, 16(1), n. p.

Contreras, J. M., Estrada, A., Díaz, C. \& Batanero, C. (2010). Dificultades de futuros profesores en la 
lectura y cálculo de probabilidades en tablas de doble entrada. In M. M. Moreno, A. Estrada, J. Carrillo \& T. A. Sierra, (Eds.), Investigación en educación matemática XIV (pp. 271 280). Lleida: Sociedad Española de Investigación en Educación Matemática, SEIEM.

Correia, P. F. \& Fernandes, J. A. (2013). Caracterização das intuições de alunos do 9o ano em independência e probabilidade condicionada. In J. A. Fernandes, F. Viseu, M. H. Martinho \& P. F. Correia (Orgs.), Atas do III Encontro de Probabilidades e Estatística na Escola (pp.47-68). Braga: Centro de Investigação em Educação da Universidade do Minho.

Estrada, A., \& Díaz, C. (2006). Computing probabilities from two way tables: an exploration study with future teachers. Em A. Rossman \& B. Chance (Eds.), Proceedings of Seventh International Conference on Teaching of Statistics. Salvador (Bahia): International Association for Statistical Education.

Fernandes, J. A. (2018). Probabilidade de acontecimentos envolvendo aspetos lógicos. Bolema, 32(60), 172-190.

Fernandes, J. A., \& Gea, M. M. (2018). Conhecimento de futuros professores dos primeiros anos escolares para ensinar probabilidades. Avances de Investigación en Educación Matemática, $14,15-30$.

Fernandes, J. A., Batanero, C., Correia, P. F., \& Gea, M. M. (2014). Desempenho em probabilidade condicionada e probabilidade conjunta de futuros professores do ensino básico. Quadrante, 23(1), 43-61.

Fernandes, J. A., Correia, P. F., \& Contreras, J. M. (2013). Ideias intuitivas de alunos do 9. ano em probabilidade condicionada e probabilidade conjunta. Avances de Investigación en Educación Matemática, 4, 5-26.

Fernandes, J. A., Viseu, F., \& Gea, M. M. (2016). O conhecimento de Probabilidades de futuros educadores e professores dos primeiros anos. In L. G. W. Coan \& M. T. Moretti (Orgs.), Aplicações matemáticas com Tecnologias de Informação e Comunicação (pp. 123-142). Florianópolis, SC: Editora Insular.

Gigerenzer, G., \& Hoffrage, U. (1995). How to Improve Bayesian Reasoning Without Instruction: Frequency Formats. Psychological Review, 102(4),684-704.

Godino, J. D. (2009). Categorías de análisis de los conocimientos del profesor de matemáticas. UNIÓN - Revista Iberoamericana de Educación Matemática, 20, 13-31.

Godino, J. D., Batanero, C., \& Font, V. (2007). The onto-semiotic approach to research in mathematics education. ZDM - The International Journal on Mathematics Education, 39(1/2), 127-135.

Hill, H. C., Ball, D. L., \& Schilling, S. G. (2008). Unpacking pedagogical content knowledge: conceptualizing and measuring teachers' topic specific knowledge of students. Journal for Research in Mathematics Education, 39(4), 372-400. 
Hoffrage, U., Gigerenzer, G., Krauss, S., \& Martignon, L. (2002). Representation facilitates reasoning: what natural frequencies are and what they are not. Cognition, 84 (2002) 343-352.

Ministério da Educação e Ciência (2014). Programa de Matemática A - Ensino Secundário. Lisboa: Autor.

Ministério da Educação e Ciência. (2013). Programa de matemática para o ensino básico. Lisboa: Autor.

Polaki, M.V. (2005). Dealing with compound events. Em G.A. Jones (Ed.), Exploring probability in school: challenges for teaching and learning (pp. 191-214). Nova lorque: Springer.

Pollatsek, A., Well, A.D., Konold, C., \& Hardiman, P. (1987). Understanding conditional probabilities. Organitation, Behavior and Human Decision Processes, 40, 255-269.

Shulman, L. (1986). Those who understand: knowledge growth in teaching. Educational Researcher, 15(2), 4-14.

Tarr, J.E., \& Lannin, J.K. (2005). How can teachers build notions of conditional probability and independence? Em G.A. Jones (Ed.), Exploring probability in school: challenges for teaching and learning (pp. 215-238). Nova lorque: Springer.

Vásquez, C., \& Alsina, A. (2019). Conocimiento especializado del profesorado de educación básica para la enseñanza de la probabilidad. Profesorado. Revista de Currículum y Formación de Profesorado, 23(1), 393-419.

Watson, J.M., \& Moritz, J.B. (2002). School students' reasoning about conjunction and conditional events. International Journal of Mathematical Education in Science and Technology, 33(1), 5984. 